Research Article

\title{
Awareness and Perception on Antibiotics and Antimicrobial Resistance: A Questionnaire Based Study.
}

\author{
M. Rangapriya*, Alina Lorance, Anisha Merin Varghese, Arshi Hanif, Aruna S \\ Department of Pharmacy Practice, Swamy Vivekanandha College of Pharmacy, Elayampalayam, Namakkal, Tamilnadu, India. \\ *Corresponding author's E-mail: priyanarayan97@gmail.com
}

Received: 14-08-2020; Revised: 19-10-2020; Accepted: 27-10-2020; Published on: 15-11-2020. \begin{abstract}
Antibiotics have played a colossal role in managing numerous infectious diseases since their discovery. But today the reckless emergence of AMR and exploitation of antibiotics are of pronounced alarm. Humankind is drifting into an era of multiple drug resistance and an urgent action is required to halt the advent of post-antibiotic era. It is in this regard that the study is undertaken and performed. The aim of this study is to assess the knowledge, attitude and practice of general public centered on antibiotics usage and its resistance. A descriptive study among 516 subjects following with simple random sample selection was conducted between May and July 2020 and was done using a structured questionnaire. The data was analyzed using ANOVA (SPSS VERSION 21.0). Out of 516 respondents, majority has agreed the use of antibiotics in viral infections. About $2 / 3 \mathrm{rd}$ of the population has answered that antibiotics can be taken in cold and cough. About $80 \%$ of the population think there is misuse of antibiotics. Around $89 \%$ of respondents consult a physician prior to initiate antimicrobial therapy. Our study reveals that the respondents had moderate knowledge and a basic attitude regarding prudent use of antibiotics. Thereby, creating awareness and educating people is significant in the rational use of antibiotics and to minimize the adversities.
\end{abstract}

Keywords: Antibiotics, Resistance, Knowledge, Attitude, Practice.

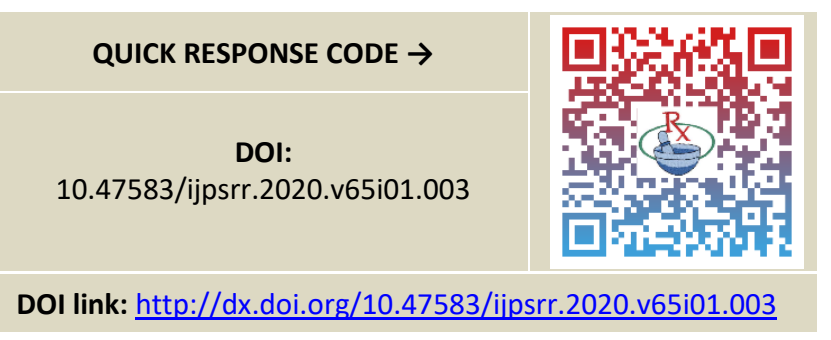

\section{INTRODUCTION}

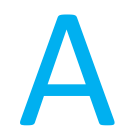

ntibiotics have a profound impact on the management of infectious diseases and are an essential aspect of modern healthcare practice. Bacteria with susceptible phenotype to antibiotics are inhibited or killed through bacteriostatic or bactericidal method. The administration of this life saving drug is increasing drastically worldwide to treat the infections making them an indispensable form of medical treatment. With the constant selective pressure or environmental pressure, bacteria thrive to survive the condition by evolving themselves. With this evolution they develop resistant phenotype and in a matter of few seconds millions of daughter cells are produced due to their higher replication rate and becomes predominant. Each time an antibiotic is inappropriately administered, we are introducing an evolutionary gravity that speeds up the spread of antibiotic resistant bacteria or antimicrobial resistance (AMR), posing a substantial rise in morbidity, mortality and decline in quality of life (QoL), making it one 1. of the world's most fatal health crises ${ }^{2}$. India is in the 1. highest risk of becoming the AMR capital of the world, 2 . owing to approximately 50,000 neonatal deaths due to developing resistance of antimicrobials ${ }^{8}$.
As per the report by UN Interagency Coordination Group 2019, the death due to AMR could increase to 10 million deaths globally per year by 2050 if no prompt action is taken. The problem arises when there is indiscriminate use of antibiotics in healthcare practice as well as in public which makes it multi-faceted problem. In many areas, the easy availability of antibiotics over the counter has escalated AMR by one step ahead. Multicountry report by World Health Organization [WHO], 2015 states that awareness of the existence of antibiotic resistance was widespread in general populations but understanding of the implications was mixed ${ }^{4}$. Thus, bringing changes to these areas can bridge the gap between knowledge, attitude and practice (KAP) regarding antibiotic use in public.

Hence this study aims to collect information constructed on knowledge, attitude and practice of the general population on the usage of antibiotics and its resistance. The current study data provides a better understanding on general population views on antibiotic usage and its resistance that can provide us the route map where the mishandling happens with antibiotics and the areas that promote the AMR. This information could be an efficient tool in assessment and providing the baseline for the development of comprehensive and effective educational interventions for future perspective to re-mediate the gap in KAP is the expected outcome of the study. 


\section{METHODOLOGY}

\section{Study Setting and Design}

This study was a descriptive conducted among general population following simple random sample selection. The self- administered survey was conducted for a period of 3 months May- July 2020.

\section{Target Population}

The study population consisted of general population covering both medical and non-medical disciplines. The questionnaires were distributed to the participants after obtaining their informed consent. Only completely filled questionnaires were included in the study for final analysis.

\section{Survey Tool Development, Pre-Testing, and Validation}

On referring to previous similar KAP studies concerning antibiotic use in the literature, the structured selfadministered questionnaire was developed based on the studies carried out in China, India, Saudi and Nepal with similar objectives ${ }^{5-8}$. It was designed and adapted to cover all the main key points of the research and in a way that suited the general population. The questionnaire was then reviewed and assessed by three clinical pharmacy professors at Swamy Vivekanandha College of Pharmacy for its content relevance and appropriateness (figure1)

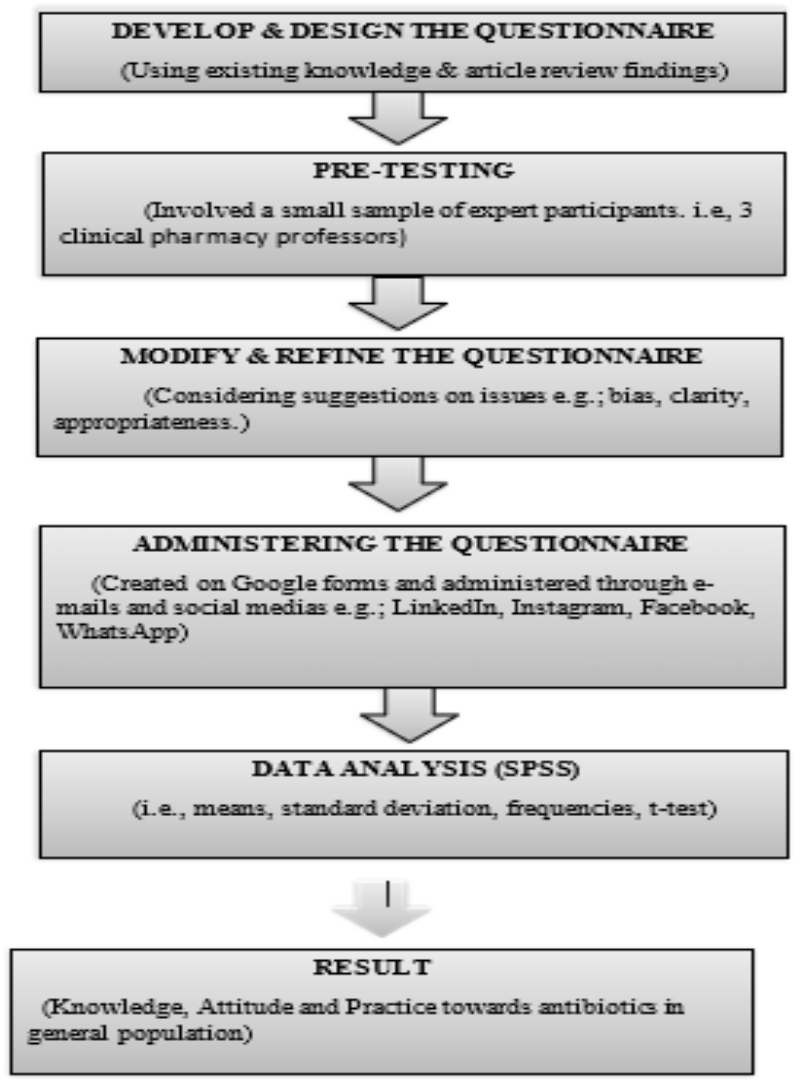

Figure 1: Methodology of the present study

\section{Research-Instrument Section and Scoring System}

A 23-point self-administered questionnaire containing both open and closed ended questions was developed pertaining to the responders' knowledge, attitude and practices regarding antibiotic use and self-medication.

The first part pertained to a collection of demographic information of the students: Age, gender and profession.

The questions in the second part of the questionnaire assessed the students" knowledge containing 9 closedended questions about antibiotic resistance and usage. The participants" knowledge was assessed by using a set of true and false questions.

The third part of the questionnaire consisting of 8 closedended questions addressed their attitude related to use of antimicrobials and its resistance

The fourth part of the questionnaire containing 6 both open and close-ended questions related to their selfreported practices regarding antibiotic usage.

Questions evaluating knowledge, attitude and practices of antibiotic use were associated with categorical responses: (yes/no). Each correct answer was scored 1 point, while a wrong answer was given 0 points making minimum 0 and maximum 23 points for each participant.

The above-mentioned questionnaire was administered by two different approaches. The questionnaire was created on Google forms and the link was provided to the respondents through e-mails and social Medias (LinkedIn, Instagram, Facebook, WhatsApp).

\section{Data Analysis}

The statistical analyses of categorical variables (such as responses to questions on knowledge, attitude and practice) were done using Chi square test used to compare differences in the proportions of qualitative variables. All test results were interpreted for statistical significance using a threshold of $p=0.05$. Spearman correlation test was applied to estimate the relations of knowledge, attitude and practice. All the analyses were done using ANOVA (SPSS VERSION 21.0).

\section{RESULT}

\section{Characteristics of the study population}

The study participants were 516 subjects and response rate were $100 \%$. Among these respondents, the majority were females of $64.7 \%$ with males of $35.3 \%$. The age group was predominantly between 18- 29 years old. The majority of the sample participants $(58.7 \%)$ were related to medical profession (MP) or medical education and minority of $41.3 \%$ were non-medical related profession (NMP) or education. 
Table 1: Knowledge on antibiotic use and resistance

\begin{tabular}{|c|c|c|c|c|}
\hline SL NO & QUESTIONS (CORRECT ANSWER) & TOTAL \%(n) & CHI SQUARE & p-VALUE \\
\hline K1 & Are there any bacteria in human body which can be helpful for us? (YES) & $4(21)$ & 0.8 & 0.03 \\
\hline K2 & Can antibiotics be used to cure infections caused by bacteria? (YES) & $30(155)$ & 4.05 & 0.05 \\
\hline K3 & Can antibiotics be used to cure infections caused by virus? (NO) & $50(26)$ & 5.21 & 0.06 \\
\hline K4 & $\begin{array}{l}\text { Is it okay to stop taking antibiotics without finishing complete dose if you } \\
\text { are feeling well? (NO) }\end{array}$ & $26(110)$ & 9.8 & 0.32 \\
\hline K5 & Should chest infection always be treated with antibiotics? (NO) & $7(33)$ & 14.07 & 0.41 \\
\hline K6 & Can antibiotics speed up the recovery process of cold and cough? (NO) & $8(42)$ & 18.7 & 0.08 \\
\hline K7 & Can antibiotics cause any side effects? (YES) & $10(67)$ & 4.5 & 0.51 \\
\hline K8 & Have you heard the term "Antibiotic Resistance"? (YES) & $8(41)$ & 6.7 & 0.62 \\
\hline K9 & $\begin{array}{l}\text { Do you think frequent use of antibiotics can decrease occurrence of } \\
\text { infection? (NO) }\end{array}$ & $4(21)$ & 8.1 & 0.07 \\
\hline
\end{tabular}

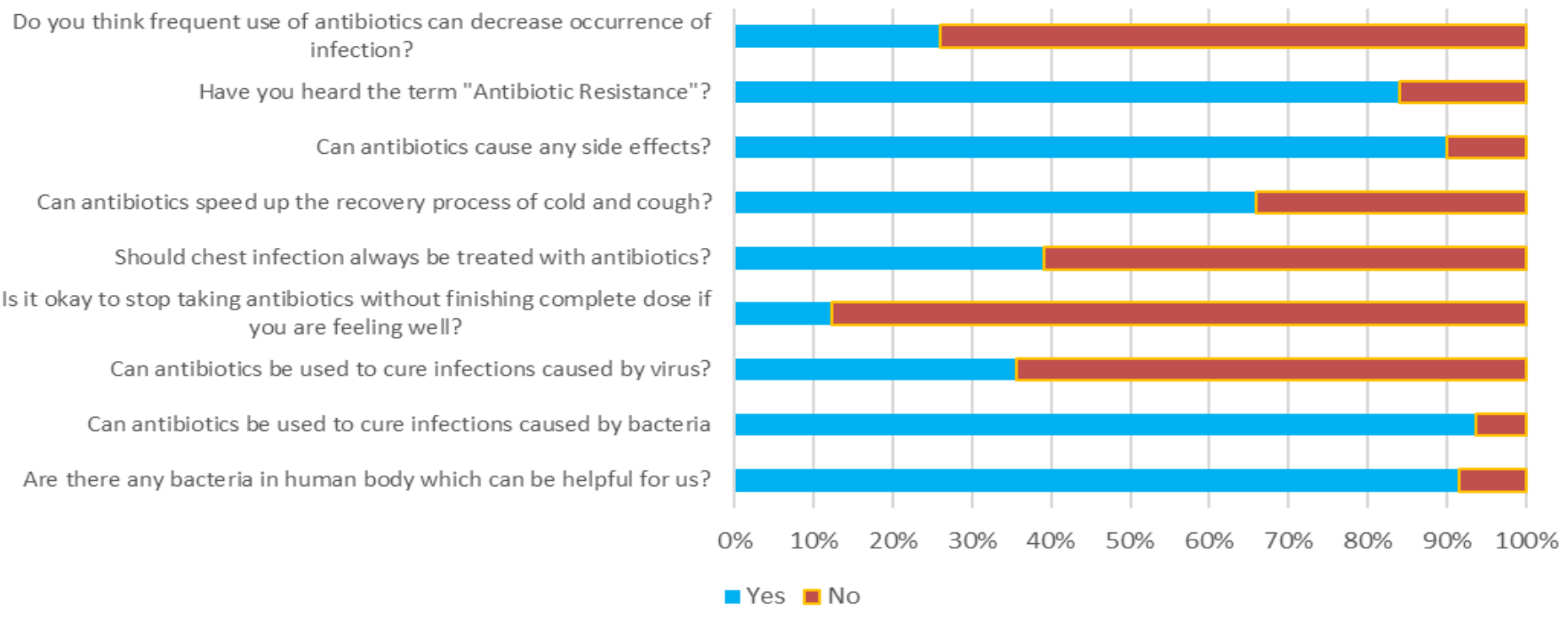

Figure 2: Responses to Questions Related to Knowledge in Relation to Antibiotic Use and Resistance

\section{Knowledge Level of Antibiotic Use and its resistance}

The majority of the respondents knew the existence of bacteria that contributes to the better health of a person (91.5\%) and were also aware antibiotics treats bacterial infection (93.6\%) as in shown in responses to questions related to knowledge in relation to antibiotic use and resistance (figure 2). Comparatively, the respondents didn't show a higher percentage in answering correctly to K3. The findings of participants answering to K3 shows about $50 \%$ of population under non-medical related healthcare/ work and $25 \%$ of medical related population answered incorrectly. About 2/3rd respondents have answered antibiotics can be taken for cold and cough, $77 \%$ belonged to non-medical related sample and interestingly $58 \%$ medical related. The study respondents have stated antibiotics can be taken for chest infection in which $43 \%$ belong to medical profession and $33 \%$ to non-medical field as shown in knowledge on antibiotic use and resistance (table 1 ). K4 shows $88 \%$ of sample population has medication adherence to the prescription given to them. On an average, healthcare associated work/ education population has secured $80.4 \%$ and $68.6 \%$ by non-medical related population. Both the category of population has lower percentage in K3, K5 and $\mathrm{K} 6$ questions due to the confusion that arises between bacterial and viral infections as in most cases are of viral etiology.

Do you think frequent use of antibiotics can decrease occurrence of infection?

Have you heard the term "Antibiotic Resistance"?

Can antibiotics cause any side effects?

Can antibiotics speed up the recovery process of cold and cough?

Should chest infection always be treated with antibiotics? Is it okay to stop taking antibiotics without finishing complete do self you are feeling well?

Can antibiotics be used to cure infections caused by virus? Can antibiotics be used to cure infections caused by bacteria?

Are there any bacteria in human body which can be helpful for us?

\section{Attitude level of antibiotic use}

About $80 \%$ of study participants is aware antibiotics are misused and can lead to AMR if not necessary actions are taken to flatten the curve. Amongst this on average, $21.5 \%$ of medical and $26.5 \%$ non-medical related participants 
didn't approve antibiotics and its resistance is a concerned matter. Though the study population is aware of the issue, only $50 \%$ knew it can create an impact in one's self-health and family health. Where as the $55 \%$ of non-medical and $42 \%$ of medical field sample believed it doesn't create an effect in one's health. $59.74 \%$ (306) of the population preferred antibiotics for chest infection as in attitude of study subjects on antibiotic use and resistance (table 2). In Contradict $92 \%$ acknowledges it is necessary to know the appropriate use of antibiotic as shown in responses to questions related to attitude in relation to antibiotic use and resistance (figure 3 ). Overall, on average scale the medical related population scored higher (84.4\%) than non-medical related with $63.8 \%$.

Table 2: Attitude of study subjects on Antibiotic use and resistance

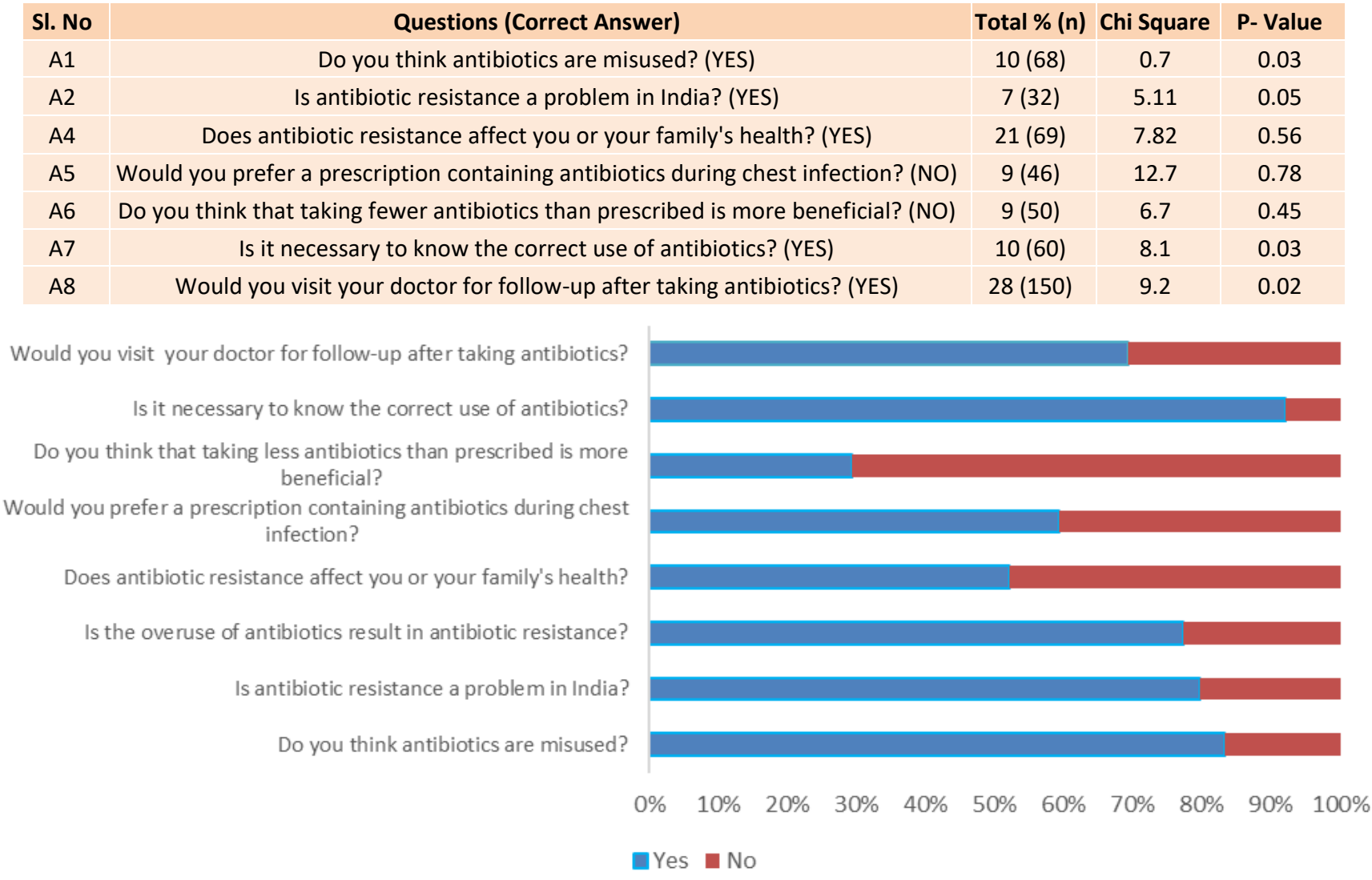

Figure 3: Responses to Questions Related to Attitude in Relation to Antibiotic Use and Resistance

Would you visit your doctor for follow-up after taking antibiotics?

Is it necessary to know the correct use of antibiotics?

Do you think that taking less antibiotics than prescribed is more beneficial?

Would you prefer a prescription containing antibiotics during chest infection?

Does antibiotic resistance affect you or your family's health? Is the overuse of antibiotics result in antibiotic resistance? Is antibiotic resistance a problem in India?

Do you think antibiotics are misused?

\section{Practice level of antibiotic use}

Nearly $90 \%$ of study respondents reported of consulting the physician prior to antibiotic administration as shown in responses to questions related to practice in relation to antibiotic use and resistance (figure 4). Whereas 60\% stated of not purchasing antibiotic drug from medical store, 203 respondents were favoring the statement in which $46 \%$ belonged to non-medical field and $33 \%$ were medical related sample population. Amongst the 147 (30\%) respondents who stated that they share the antibiotic medication with their family/ friends, 30\% were non-medical related population and $27 \%$, were medical related population (table 3 ). $82 \%$ reported to complete the duration course of antibiotic prescription. On average scale for practice, non-medical related population scored $72.7 \%$ and medical population with $79 \%$.

The statistical analysis which has been performed for this study is to exhibit a correlation between the various parameters which are being studied (Table 4). There was a statistically significant difference between the Mean knowledge score and four different characteristics; namely; age, occupation, income, education (table 5-7) with $\mathrm{p}<0.05$.

Relationship between knowledge, attitudes and practices

Spearman rank order correlation revealed a positive association between each pair of the knowledge, attitude and practice scores for respondents as shown in the table 4. 
Table 3: Practice about antibiotic usage and resistance among study subjects

\begin{tabular}{|c|c|c|c|c|}
\hline SL. NO & Questions (Correct Answer) & Total \% (n) & Chi-square & P- Value \\
\hline P1 & Do you consult a doctor before starting an antibiotic? (YES) & $12(72)$ & 0.93 & 0.41 \\
\hline P2 & We can buy antibiotic from medicine shops/ pharmacies directly. (NO) & $8(83)$ & 8.12 & 0.03 \\
\hline P3 & We can use antibiotics after the suggestions from friends/neighbors. (NO) & $27(121)$ & 9.27 & 0.65 \\
\hline P4 & Do you follow the advertisement (leaflets/ internet etc.) while purchasing & $15(93)$ & 7.12 & 0.32 \\
\hline P5 & antibiotics? (NO) & $19(89)$ & 6.78 & 0.05 \\
\hline P6 & Do you share antibiotics with your friends/ family members if they get sick? (NO) & $14(100)$ & 4.36 & 0.07 \\
\hline
\end{tabular}

Do you stop taking antibiotic without completing full course?

Do you share antibiotics with your friends/ family members if they get sick?

Do you follow the advertisement (leaflets/internet etc) while purchasing antibiotics?

We can use antibiotics after the suggestions from friends/neighbours.

We can buy antibiotic from medicine shops/ pharmacies directly.

Do you consult a doctor before starting an antibiotic?
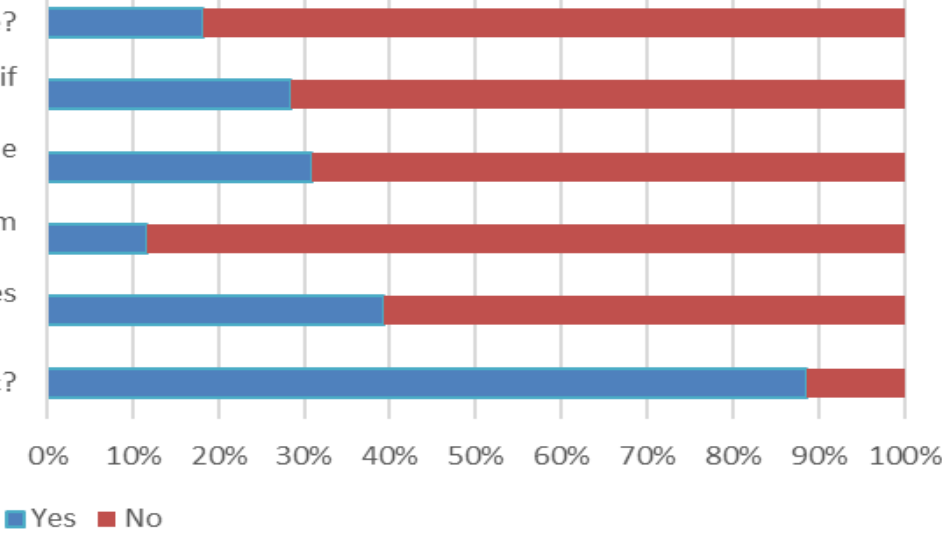

Figure 4: Responses to Questions Related to Practices in Relation to Antibiotic Use

Table 4: Spearman Rank Correlation Between Knowledge, Attitude and Practice

\begin{tabular}{|c|c|c|}
\hline Variable & Correlation Coefficient & p-Value \\
\hline Knowledge-attitude & 0.06 & 0.016 \\
\hline Knowledge-practice & 0.04 & 0.021 \\
\hline Attitude- practice & 0.21 & 0.04 \\
\hline
\end{tabular}

Table 5: Overall Knowledge, Attitude and Practice Mean Scores Vs Gender

\begin{tabular}{|c|c|c|c|c|c|c|}
\hline \multirow{2}{*}{ Variable } & \multicolumn{2}{|c|}{ Knowledge Score } & \multicolumn{2}{c|}{ Attitude Score } & \multicolumn{2}{c|}{ Practice Score } \\
\cline { 2 - 6 } & Mean \pm SD & P-value & Mean \pm SD & P-value & Mean \pm SD & P-value \\
\hline MALE & $22 \pm 0.01$ & 0.03 & $81 \pm 0.01$ & 0.08 & $43 \pm 0.01$ & 0.06 \\
\hline FEMALE & $35 \pm 0.01$ & 0.06 & $66 \pm 0.01$ & 0.10 & $94 \pm 0.01$ & 0.04 \\
\hline
\end{tabular}

*For Incorrect Answer- 0 Point and Correct Answer-1 Point. * Source: Compiled By Author

Table 6: Overall Knowledge, Attitude and Practice Mean Scores Vs Age Group

\begin{tabular}{|c|c|c|c|c|c|c|}
\hline Variable & \multicolumn{3}{|c|}{ Knowledge Score } & \multicolumn{2}{c|}{ Attitude Score } & \multicolumn{2}{c|}{ Practice Score } \\
\hline $18-29$ & $84 \pm 0.03$ & 0.07 & $74 \pm 0.03$ & 0.05 & $41 \pm 0.01$ & 0.52 \\
\hline $30-44$ & $40 \pm 0.03$ & 0.06 & $81 \pm 0.03$ & 0.07 & $32 \pm 0.01$ & 0.03 \\
\hline $45-59$ & $20 \pm 0.03$ & 0.51 & $40 \pm 0.03$ & 0.03 & $50 \pm 0.01$ & 0.05 \\
\hline$>=60$ & $15 \pm 0.03$ & 0.02 & $20 \pm 0.03$ & 0.41 & $18 \pm 0.01$ & 0.42 \\
\hline
\end{tabular}

*For Incorrect Answer- 0 Point and Correct Answer-1 Point. * Source: Compiled by Author

Table 7: Overall Knowledge, Attitude and Practice Mean Scores Vs Healthcare Education

\begin{tabular}{|c|c|c|c|c|c|c|}
\hline Variable & \multicolumn{2}{|c|}{ Knowledge Score } & \multicolumn{2}{|c|}{ Attitude Score } & \multicolumn{2}{|c|}{ Practice Score } \\
\hline \multicolumn{7}{|c|}{ Healthcare Work/ Education } \\
\hline YES & $45 \pm 0.01$ & 0.034 & $71 \pm 0.01$ & 0.04 & $45 \pm 0.01$ & 0.02 \\
\hline NO & $54 \pm 0.01$ & 0.021 & $82 \pm 0.01$ & 0.021 & $33 \pm 0.01$ & 0.54 \\
\hline
\end{tabular}

*For Incorrect Answer- 0 Point and Correct Answer-1 Point. * Source: Compiled by Author 


\section{DISCUSSION}

The majority of population were aware of antimicrobial resistance is a global concern as well as nationwide problem. Based on survey, majority of the population knew about the presence of beneficial bacteria in our body and antibiotics are used to cure infection caused by bacteria that they are clearly known and they accept. Contradictory, as per the KAP survey, it shows antimicrobials are used in discriminately, meaning they have less knowledge on the significance of antibiotics in viral infections assuming bacterial etiology. Half of the sample population believes that antibiotics can cure the viral etiological infections, indirectly showing there is confusion among the general population on the basic knowledge of antibiotics and causative agents of common illness. The results of our study show that knowledge of when antibiotics should be used as well as the risk of antibiotic resistance is poor. We interpret this as an indication that the term resistance and an acceptance of its severity have reached the general public even though the biological mechanisms behind it remain largely unknown. Such wrong disbeliefs can lead to higher selfconsumption of antibiotics which can result in a corresponding increase in the bacterial resistance.

Study base resting on attitude, nearly $90 \%$ is aware of the antibiotics misuse. The majority of the participants were expressive that over use of antibiotics will lead to antibiotic resistance. The participants showed good medication adherence and follow-up as and when it is required. All these indicate a positive attitude. However, only $50 \%$ were aware that AMR could create an impact in one's health and to their family.

There is good amount of practice score in respondents. There is a positive practice when it comes to not sharing the medication with their family/ friends. A majority of the population has always consulted a doctor before starting an antibiotic and most of them always completed the full course of the prescribed treatment. Whereas $40 \%$ states they have purchased the antibiotics from an outlet store. There isn't a proper control in dispensing the Over the Counter (OTC) dispensing of antibiotics without prescription. The antibiotics are now dispensed everywhere, any time without a prescription, apart from this indiscriminate use of antibiotics in health care settings is escalating the issue. The results obtained from the overall survey shows the awareness of AMR and proper medication adherence in the population is prevalent. But KAP on basics of antibiotics are majorly deficient. This study acts as a tool for future interventions when providing well-organized, strategic and designed information for the general public in collaboration with the whole team of health care professionals in raising antibiotic literacy and promotion on health.

\section{CONCLUSION}

The present study shows that, respondents are well aware about AMR but are heedless of the consequences. Participants displayed poor knowledge and attitude particularly in regards with the use of antibiotics in viral infections, the role of antibiotics in treating cold, cough and chest infections. They also share a great deal of confusion, whether AMR will affect their family or not. We found that respondents have fairly good practices including self-medication and sharing of antibiotics. Hence, battling the resistance bugs mandates the prudent use of antibiotics which requires appropriate awareness and education about the safe use of antibiotics. The data from our findings could serve as a baseline for development and monitoring of future interventions. There is a high need for coordinated actions such as public education measures, patient education and counselling for judicious use of antibiotics among community members.

Acknowledgment: The authors would like to thank all the respondents for their inestimable participation in this study.

\section{REFERENCES}

1. Zain A. Unregulated antibiotic use could kill 10 million people by 2050.2018. Available from: https://www.khaleejtimes.com/news/uae-health//unregulatedantibiotic-use-couldkill10-million- people-by-2050. Accessed July 6,2017.

2. Lakminarayan R. Matsoso P. Pant S, Brower C, Rottingen JA et al. Assess to effective antimicrobials: a worldwide challenge. Lancet 387, 2016, 168-75.

3. 4- World Health Organization [WHO] Antibiotic Resistance: MultiCountry Public Awareness Survey. Geneva: WHO Press, 2015.

4. Huang, Y.; Gu, J.; Zhang, M. Knowledge, attitude and practice of antibiotics: A questionnaire study among 2500 Chinese students. BMC Med. Educ. 13, 2013, 163

5. Khan, A.K.A, Banu, G.K.K.R. Antibiotic Resistance and Usage-A Survey on the Knowledge, Attitude, Perceptions and Practices among the Medical Students of a Southern Indian Teaching Hospital. J. Clin. Diagn. Res. 7,2013, 1613-1616.

6. Minen, M.T.; Duquaine, Marx, M.A. A survey of knowledge, attitudes, and beliefs of medical students concerning antimicrobial use and resistance. Microb. Drug Resist. 16,2010, 285-289.

7. Poonam Shah, Rajeev Shrestha, Zongfu Mao, Yilin Chen, Yan Chen. Knowledge, Attitude, and Practice Associated with Antibiotic Use among University Students: A Survey in Nepal. Int. J. Environ. Res. Public Health 2019, 16, 3996; doi:10.3390/ijerph1620399

8. Chaudhry D, Tomar p. Antimicrobial Resistance: the next big pandemic. Int J Community Med Public Health 4, 2017, 2632-6.

Source of Support: None declared.

Conflict of Interest: None declared.

For any question relates to this article, please reach us at: editor@globalresearchonline.net

New manuscripts for publication can be submitted at: submit@globalresearchonline.net and submit_ijpsrr@rediffmail.com 